Document downloaded from:

http://hdl.handle.net/10251/176616

This paper must be cited as:

Sánchez, MG.; Fajardo-Delgado, D.; Vidal-Gimeno, V.; Verdú Martín, GJ. (2020). A hybrid genetic algorithm to reduce the radiation dose in CR images. Radiation Physics and Chemistry. 167. https://doi.org/10.1016/j.radphyschem.2019.04.025

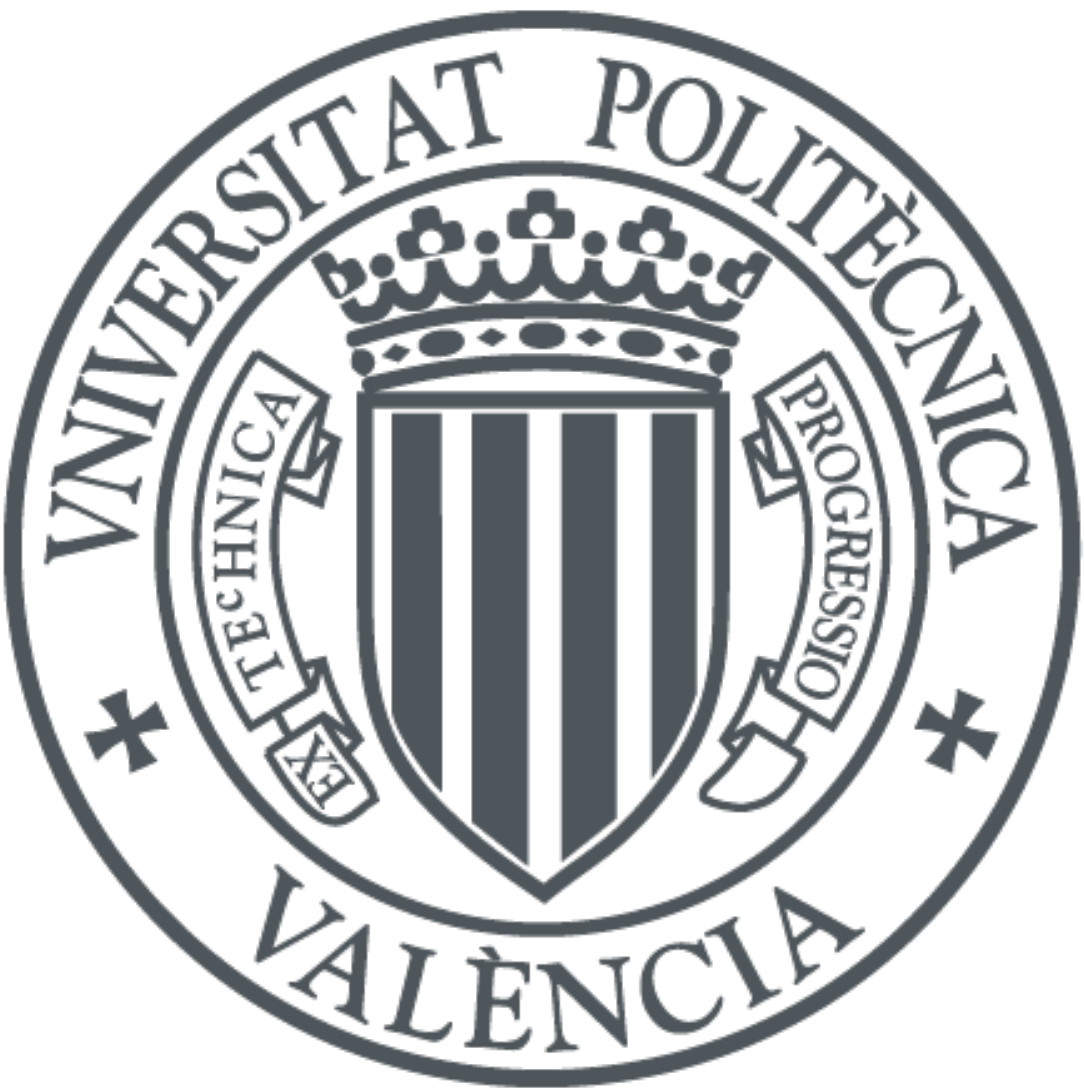

The final publication is available at

https://doi.org/10.1016/j.radphyschem.2019.04.025

Copyright Elsevier

Additional Information 


\title{
A hybrid genetic algorithm to reduce the radiation dose in CR images
}

\author{
M.G. Sánchez ${ }^{\mathrm{a}}$, D. Fajardo-Delgado ${ }^{\mathrm{a}, *}, \mathrm{~V}$. Vidal ${ }^{\mathrm{b}}$, G. Verdúc \\ ${ }^{a}$ DSyC, TecNM, Instituto Tecnológico de Cd. Guzmán, Av. Tecnológico \#100, 49100, \\ Cd. Guzmán, Jalisco, Mexico \\ ${ }^{b}$ DSIC, Universitat Politècnica de Valencia, Camino de Vera s/n, 46022, Valencia, Spain \\ ${ }^{c}$ DIQN, Universitat Politècnica de Valencia, Camino de Vera s/n, 46022, Valencia, \\ Spain
}

\begin{abstract}
The quality of computed radiography (CR) images typically relate to patient radiation exposure. The lower the X-ray dose exposure, the higher the level of inherent noise in the $\mathrm{CR}$ images. In this work, we address the noise reduction problem by using an estimation of the standard deviation of the noise as an objective function to minimize. We propose a hybrid genetic algorithm for this aim, which produces improved versions of CR images. We also applied an edge-detection method based on the Canny algorithm to preserve the edges of the original CR images. We executed our proposed algorithm for CR images obtained under different radiation exposures. Experimental results show that our solution improves lower radiation CR images reaching a quality as similar to those with higher radiation doses.
\end{abstract}

Keywords: Computed radiography, Radiation dose, Evolutionary algorithms

\section{Introduction}

Computed radiography $(\mathrm{CR})$ is a digital X-ray imaging modality based on the principle of photostimulable phosphor luminescence. CR systems use a phosphor plate, similar in appearance to a conventional film-screen, to store an X-ray image as a latent energy distribution. This plate releases the

\footnotetext{
${ }^{*}$ Corresponding author.

Email address: dfajardo@itcg.edu.mx (D. Fajardo-Delgado)
} 
trapped energy in the form of light which is converted to a digital image that is proportional to the X-ray intensity.

$\mathrm{X}$-rays produce ionizing radiation which is potentially harmful to patients. The amount of ionizing radiation transmitted in X-rays is small; however, the probability of stochastic effects increases with doses on patient. In medical diagnostic imaging, ionizing radiation exposure in patients should always be kept 'as low as reasonably achievable' (ALARA), giving the minimum amount of radioactive tracer necessary to provide a useful diagnostic test (ICRP, 1964). Additionally, the radiation dose transmitted to the patient may vary. According to Hall (2002), children are up to ten times more sensitive to ionizing radiation than adults.

The ionizing radiation exposure to a patient during a CR examination is directly proportional to an X-ray tube current, measured in milliampereseconds (mAs). Higher mAs in CR systems could tolerate a high level of over-exposure without image quality degradation (Ma et al., 2013). However, if the exposure factors and radiation dose are too low, the resulting images can appear noisy due to photon deficiency (Kei et al., 2014). Therefore, the tradeoff between radiation dose and image quality is critical.

Noise is one of the primary factors that affect the quality of a CR image. However, only a few papers have studied the relationship between noise in CR images and X-ray tube current (mAs) settings. Juste et al. (2008) validate a technique to evaluate the similarity between CR images perturbed by Gaussian noise and their corresponding versions obtained with a lower radiation dose. Based on this study, Sánchez et al. (2014) uses a nonlinear diffusion filter (NDF) to reduce Gaussian noise in CR images acquired with a low radiation dose. They conclude that it is possible to reduce the radiation dose in patients by decreasing the radiation exposure (mAs) and then filtering the image with the NDF. Similar studies of noise in Computed Tomography can be found in (Li et al., 2002; Flores et al., 2016; Parcero et al., 2017).

In this paper, we address the noise reduction problem in lower radiation CR images. Our motivation is to produce images with a quality similar to those obtained through higher radiation doses. Our work is inspired by the approaches proposed by Sánchez et al. (2014) and Juste et al. (2008) but we address the problem from a different perspective. We use the standard deviation of the noise in $\mathrm{CR}$ images as an objective function to minimize through an evolutionary approach. We propose a hybrid genetic algorithm which integrates the execution of two filters and combine their results to improve them. 
We performed experimental simulations to compare the resulting images of the proposed algorithm with CR images with higher radiation doses.

We organize the paper as follows. Section 2 explains our methodology and describes the proposed hybrid genetic algorithm. Section 3 discusses the results of the experimental study. Finally, Section 4 presents some concluding remarks.

\section{Methodology}

The methodology is as follows. First, we compute the standard deviation of the noise in the CR image. Then, we use this value to estimate the radiation dose that was employed to generate the $\mathrm{CR}$ image. Later, we use a hybrid genetic algorithm which seeks to minimize the standard deviation of the noise in the CR image. Finally, we compare the resulting image of the algorithm against a CR image obtained with a higher radiation dose.

\subsection{The standard deviation of noise}

In this work, we use the total variation minimization framework of Rudin et al. (1992), for which a diversity of variational methods for image restoration has been proposed (Guidotti and Lambers, 2009; Hajiaboli, 2011; Puvanathasan and Bizheva, 2009). In particular, we consider the following equation as described in (Rudin et al., 1992; Vogel and Oman, 1996; Weickert et al., 1998), which have proved to be suitable for medical imaging:

$$
J(u, \beta, c, \epsilon)=\int_{\Omega}\left(\sqrt{\beta^{2}+\|\vec{\nabla} u\|^{2}}+\frac{c}{2}\left(u-I_{o}\right)^{2}+\frac{\epsilon}{2}(\vec{\nabla} u)^{2}\right) d \vec{x},
$$

where $I_{o}$ denotes the observed image (perturbed by noise), $u$ is the desired true solution (a denoised version of $I_{o}$ ), $\beta$ is RRRRRR, $c$ is a constant that represents RRRRRR, $\epsilon$ represents the error value, and $\Omega$ is a convex region in $\mathbb{R}^{2}$ which constitutes the support space of the surface area $u(x, y)$ of the image. When $\beta=1$, the first term of the equation represents the surface area of the image, while $\beta=0$ gives the total variation of $u$ (Vogel and Oman, 1996). The second term denotes the distance between the observed image and the desirable solution. Finally, the last term controls the regularity of the solution.

The noise reduction problem corresponds to the following constrained optimization problem: 


$$
\begin{array}{ll}
\min _{u} & J(u, \beta, c, \epsilon) \\
\text { subject to: } & \frac{\int_{\Omega}\left(u-I_{o}\right)^{2} d \vec{x}}{\int_{\Omega} d \vec{x}}=\sigma^{2},
\end{array}
$$

where $\sigma$ denotes the standard deviation of the noise, which describes the magnitude of the error $\epsilon$. The parameter $\sigma$ is a priori unknown, so we use a robust estimation of the standard deviation of the noise proposed by Donoho (1995) and expressed as follows:

$$
\sigma=\frac{\operatorname{median}\left(\left|D_{i j}\right|\right)}{0.6475}
$$

where $D_{i j}$ represent the diagonal coefficients of Daubechies wavelet of order 25.

Next, we describe our proposed hybrid genetic algorithm.

\subsection{The hybrid genetic algorithm}

The proposed hybrid genetic algorithm consists of a modified version of the algorithms presented in (Paiva et al., 2015) and (Fajardo-Delgado et al., 2016). This algorithm takes as input a CR image $I_{o}$, and returns as output an improved version of $I_{o}$ (with a lower standard deviation of the noise). The algorithm uses a Gaussian low-pass filter and the fuzzy metric peer group filter (FMPGF) (Camarena et al., 2008) to produce denoised images of $I_{o}$, denoted as $I_{G}$ and $I_{F}$, respectively. These images are merged to integrate them as 'individuals' of an evolving population. In this context, each individual represents a CR image that characterizes a version of $I_{o}$, and a population represents a set of individuals. Hereinafter, we will use 'image' and 'individual' interchangeably.

The general procedure for the hybrid genetic algorithm consists of the following steps:

1. (Initialization) Execute filters Gaussian low-pass and FMPGF over $I_{o}$ to generate two new images, $I_{G}$ and $I_{F}$, respectively. Then, perform a pixel recombination procedure that randomly exchanges pixels between $I_{G}$ and $I_{F}$ to generate an initial population of size $\mu$.

2. (Estimation) Use Eq. (3) to compute $\sigma$ (an estimation of the density of noise) for each image of the population. 
3. (Parent selection) Randomly selects, with replacement, pairs of images from the population until building a pool of $\lambda=P_{c} \mu$ 'parents', where $P_{c}$ is the recombination rate.

4. (Recombination) Recombine pairs of the selected parents to generate $\lambda$ 'offsprings'. We implemented the same recombination operators a proposed in (Fajardo-Delgado et al., 2016).

5. (Mutation) Mutate each offspring with probability $P_{m}$ through the execution of one of the filters Gaussian or FMPGF selected randomly and uniformly.

6. (Population replacement) Combine the set of images from the previous generation and their offsprings, and selects the $\mu$ images with the lowest $\sigma$ to create a new generation.

7. Repeat steps 2-6 until complete $\tau$ generations and returns the best image of the last generation.

Table 1 shows the parameters settings of the proposed algorithm. We selected the values of these parameters by the execution of preliminary trials considering the tradeoff between time and efficiency. For the case of FMPGF, we use the parameters proposed by (Camarena et al., 2008). The Gaussian low-pass filter uses the default parameters provided by Matlab.

Table 1: Configuration set for the hybrid genetic algorithm.

$\begin{array}{ll}\text { Size of the population } & \mu=30 \\ \text { Recombination rate }\left(P_{c}\right) & 0.98 \\ \text { Mutation rate }\left(P_{m}\right) & 0.3 \\ \text { Population replacement } & (\mu+\lambda) \text { selection } \\ \text { Completion criteria } & \begin{array}{l}\text { when the algorithm reaches } \tau=10 \\ \text { generations } \\ \text { FMPGF }\end{array} \\ \text { Gaussian low-pass filter } & \begin{array}{l}\text { A correlation kernel of size } 3 \times 3, \text { and } \\ \text { a standard deviation of } 0.5\end{array} \\ & \end{array}$

Finally, since filters used in the proposed algorithm may blur the edges of images, we initially extract the edges from the original CR image through the traditional Canny edge detection method (Canny, 1986). After the evolution process, we added the extracted edges to the resulting CR image. 


\section{Results and discussions}

We used the chest of a female Alderson RANDO ${ }^{\circledR}$ phantom to conduct our experiments. This phantom comprises a synthetic human skeleton embedded in a mass with properties comparable to human soft tissue. This mass is designed to have the same levels of absorption that are similar to human tissue exposed to a radiation dose. We scanned the phantom with an AGFA digital imaging device at 1, 0.8, 0.6, $0.4 \mathrm{mAs}$ using 70 and 80 kilovoltage $(\mathrm{kV})$. Fig. 1(a) shows the chest of the phantom, and Fig. 1(b) shows an example of a CR image obtained at $80 \mathrm{kV}$ and $0.4 \mathrm{mAs}$. We used a portion of CR images, with a size of $512 \times 512$ pixels, as input for the proposed hybrid genetic algorithm (HGA). We compute an estimation of the standard deviation (SD) of the output images of HGA to evaluate their similarity with a $\mathrm{CR}$ image of $1 \mathrm{mAs}$. We also compared the results of this evaluation with those obtained by a nonlinear filter (NDF) as reported in (Sánchez et al., 2014). We executed the algorithm on a $3.6 \mathrm{GHz}$ Intel Core i7 Mac, with 8 GB of RAM, and under the operating system OS X 10.14.1. The algorithm and the filters were coded in Matlab R2016b.

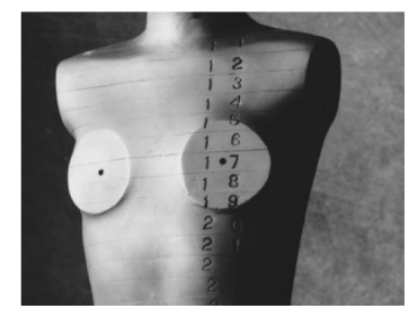

(a)

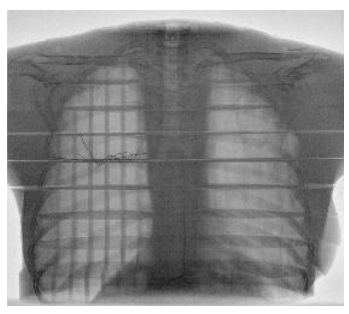

(b)

Figure 1: (a) A female Alderson RANDO phantom; (b) a CR image from the RANDO phantom at $80 \mathrm{kV}$ and $0.4 \mathrm{mAs}$.

Table 2 shows the values of SD of the noise for the resulting images at $70 \mathrm{kV}$. We observe that HGA reduces the values of SD better than NDF. Even more, the values of SD for the resulting images of HGA corresponds to $\mathrm{CR}$ images with a radiation dose higher than $1 \mathrm{mAs}$. This is not the case for the NDF. On the other hand, notice that the HGA produced an increment in the value of SD for the case of $0.6 \mathrm{mAs}$ to $0.8 \mathrm{mAs}$. We conjecture that this behavior is due to the stochastic nature of the algorithm, which uses a degree of randomness for the simulated evolution. 
Table 2: Values of standard deviation of the noise for the CR images at $70 \mathrm{kV}$.

\begin{tabular}{lrrrr}
\hline & $\mathbf{0 . 4} \mathbf{~} \mathbf{A s}$ & $\mathbf{0 . 6} \mathbf{~} \mathbf{A s}$ & $\mathbf{0 . 8} \mathbf{~ m A s}$ & $\mathbf{1} \mathbf{~ m A s}$ \\
\hline Original & 29.2156 & 24.9404 & 24.5312 & 18.4855 \\
NDF & 21.0745 & 17.9217 & 17.5932 & 13.2957 \\
HGA & 15.1916 & 12.1718 & 13.1421 & 9.4116 \\
\hline
\end{tabular}

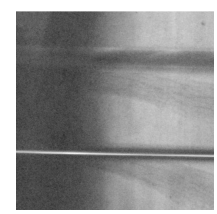

(a) $0.4 \mathrm{mAs}$

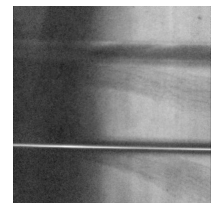

(e) $0.4 \mathrm{mAs}$

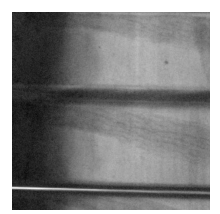

(b) $0.6 \mathrm{mAs}$

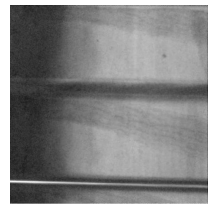

(f) $0.6 \mathrm{mAs}$

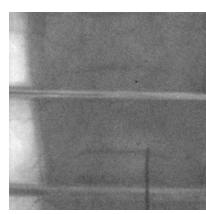

(c) $0.8 \mathrm{mAs}$

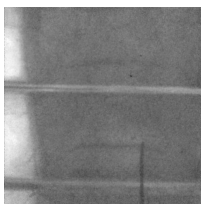

(g) $0.8 \mathrm{mAs}$

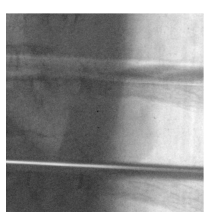

(d) $1 \mathrm{mAs}$

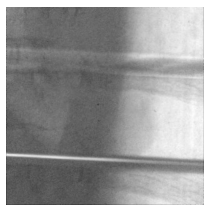

(h) $1 \mathrm{mAs}$

Figure 2: Result at 70kV; (a)-(d) original images; (e)-(h) output images of the HGA.

Table 3 shows the values of SD of the noise for the resulting images at $80 \mathrm{kV}$. These values present the same behavior as those obtained at $70 \mathrm{kV}$, in which the resulting images corresponds to $\mathrm{CR}$ images with a radiation dose higher than $1 \mathrm{mAs}$. Notice that, in general, images with a higher $\mathrm{kV}$ present less SD of noise. Figs. 2 and 3 show the resulting images after the execution of filters at $70 \mathrm{kV}$ and $80 \mathrm{kV}$, respectively.

Table 3: Values of standard deviation of the noise for the CR images at $80 \mathrm{kV}$.

\begin{tabular}{lrrrr}
\hline & $\mathbf{0 . 4} \mathbf{~} \mathbf{~ A A s}$ & $\mathbf{0 . 6} \mathbf{~ m A s}$ & $\mathbf{0 . 8} \mathbf{~ m A s}$ & $\mathbf{1} \mathbf{~} \mathbf{A s}$ \\
\hline Original & 24.0432 & 20.1867 & 19.9276 & 14.4075 \\
NDF & 17.2756 & 14.4930 & 14.3879 & 10.3120 \\
HGA & 12.8888 & 13.1171 & 11.0188 & 8.2112 \\
\hline
\end{tabular}

\section{Conclusions}

In this work, we address the reduction of radiation dose in CR images as an optimization problem. Our approach consists on applying a hybrid 


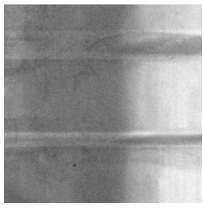

(a) $0.4 \mathrm{mAs}$

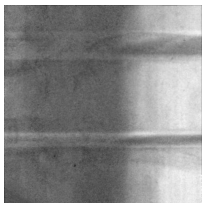

(e) $0.4 \mathrm{mAs}$

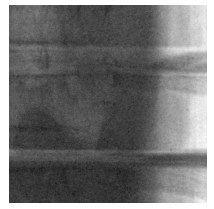

(b) $0.6 \mathrm{mAs}$

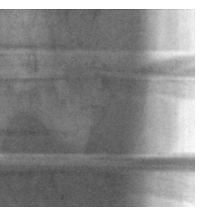

(f) $0.6 \mathrm{mAs}$

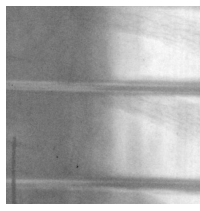

(c) $0.8 \mathrm{mAs}$

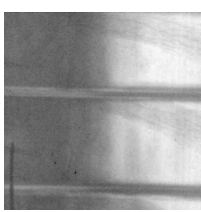

(g) $0.8 \mathrm{mAs}$

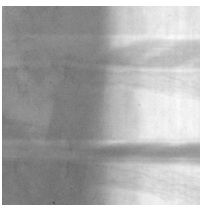

(d) $1 \mathrm{mAs}$

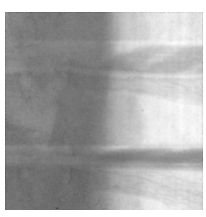

(h) $0.1 \mathrm{mAs}$

Figure 3: Result at $80 \mathrm{kV}$; (a)-(d) original images; (e)-(h) output images of the HGA.

genetic algorithm (HGA) to minimize the standard deviation (SD) of noise for a CR image obtained with low radiation exposure (mAs). Experimental results show that the proposed algorithm reduces the values of SD better than algorithms reported in the literature for the same purpose. The values of SD for the resulting images of HGA corresponds to CR images with a radiation dose higher than $1 \mathrm{mAs}$. However, it would be interesting the medical verification of our results through the evaluation of a specialized radiologist. We leave this verification as an open issue for future work. It would also be convenient to study this methodology through other optimization techniques and the use of different filters.

\section{Acknowledgments}

This work was supported by PRODEP under grant 511-6/17-8931 (ITCGUZCA-7), and by the Spanish Ministry of Economy and Competitiveness under grant TIN2015-66972-C5-4-R co-financed by FEDER funds.

\section{References}

Camarena, J.G., Gregori, V., Morillas, S., Sapena, A., 2008. Fast detection and removal of impulsive noise using peer groups and fuzzy metrics. J. Vis. Commun. Image R. 19, 20-29. doi:10.1016/j.jvcir.2007.04.003.

Canny, J., 1986. A computational approach to edge detection. IEEE T. Pattern Anal. PAMI-8, 679-698. doi:10.1109/TPAMI.1986.4767851. 
Donoho, D.L., 1995. De-noising by soft-thresholding. IEEE Trans. Inf. Theor. 41, 613-627. doi:10.1109/18.382009.

Fajardo-Delgado, D., Sánchez, M.G., Molinar-Solis, J.E., FernándezZepeda, J.A., Vidal, V., Verdú, G., 2016. A hybrid genetic algorithm for color image denoising, in: 2016 IEEE CEC, pp. 3879-3886. doi:10.1109/CEC.2016.7744281.

Flores, L., Vidal, V., Parcero, E., Verd, G., 2016. Application of a modified lsqr method for ct imaging reconstruction with low doses to patient, in: 2016 9th CISP-BMEI, pp. 1969-1974. doi:10.1109/CISPBMEI.2016.7853041.

Guidotti, P., Lambers, J.V., 2009. Two new nonlinear nonlocal diffusions for noise reduction. J. Math. Imaging Vis. 33, 25-37. doi:10.1007/s10851-0080108-z.

Hajiaboli, M.R., 2011. An anisotropic fourth-order diffusion filter for image noise removal. Int. J. Comput. Vision 92, 177-191. doi:10.1007/s11263010-0330-1.

Hall, E.J., 2002. Lessons we have learned from our children: cancer risks from diagnostic radiology. Pediatr. Radiol. 32, 700-706. doi:10.1007/s00247-0020774-8.

ICRP, 1964. Radiation protection; recommendations of the International Commission on Radiological Protection: Report of Committee IV (19531959) on Protection Against Electromagnetic Radiation Above $3 \mathrm{MeV}$ and Electrons, Neutrons and Protons. Adopted 1962, with revisions adopted in 1963. volume 4. Pergamon Press.

Juste, B., Villaescusa, J.I., Tortosa, R., Miro, R., Verdu, G., 2008. Analysis of cr dose reduction in pediatric patients, based on computer-simulated noise addition, in: IEEE EMBC, pp. 2697-2700. doi:10.1109/IEMBS.2008.4649758.

Kei, W., Hogg, P., Norton, S., 2014. Effects of kilovoltage, milliampere seconds, and focal spot size on image quality. Radiologic technology 85, 479-485. 
Li, H., Toth, T., McOlash, S., Hsieh, J., Bromberg, N., 2002. Simulating low dose ct scans by noise addition, in: 2002 IEEE Nuclear Science Symposium Conference Record, pp. 1832-1834. doi:10.1109/NSSMIC.2002.1239679.

Ma, W.K., Hogg, P., Tootell, A., Manning, D., Thomas, N., Kane, T., Kelly, J., McKenzie, M., Kitching, J., 2013. Variation of visual image quality using cr technology, relationship with e. Radiography 19, 85-86. doi:10.1016/j.radi.2012.11.007.

Paiva, J.L., Toledo, C.F., Pedrini, H., 2015. An approach based on hybrid genetic algorithm applied to image denoising problem. Appl. Soft Comput. doi:10.1016/j.asoc.2015.09.013.

Parcero, E., Flores, L., Sánchez, M., Vidal, V., Verdú, G., 2017. Impact of view reduction in ct on radiation dose for patients. Radiat. Phys. Chem. 137, 173-175. doi:10.1016/j.radphyschem.2016.01.038.

Puvanathasan, P., Bizheva, K., 2009. Interval type-ii fuzzy anisotropic diffusion algorithm for speckle noise reduction in optical coherence tomography images. Opt. Express 17, 733-746. doi:10.1364/OE.17.000733.

Rudin, L.I., Osher, S., Fatemi, E., 1992. Nonlinear total variation based noise removal algorithms. Physica D. 60, 259-268. doi:10.1016/01672789(92)90242-F.

Sánchez, M., Juste, B., Vidal, V., Verdú, G., Mayo, P., Rodenas, F., 2014. Dose reduction using non lineal diffusion and smoothing filters in computed radiography. Radiat. Phys. Chem. 95, 305-308. doi:10.1016/j.radphyschem.2013.03.017.

Vogel, C., Oman, M., 1996. Iterative methods for total variation denoising. SIAM J. Sci. Comput. 17, 227-238. doi:10.1137/0917016.

Weickert, J., Romeny, B.M.T.H., Viergever, M.A., 1998. Efficient and reliable schemes for nonlinear diffusion filtering. IEEE T. Image Process. 7, 398410. doi:10.1109/83.661190. 